\title{
Compact Model of a pH Sensor with Depletion-Mode Silicon-Nanowire Field-Effect Transistor
}

\author{
Yun Seop Yu
}

\begin{abstract}
A compact model of a depletion-mode silicon-nanowire ( $\mathrm{Si}-\mathrm{NW}$ ) pH sensor is proposed. This drain current model is obtained from the Pao-Sah integral and the continuous charge-based model, which is derived by applying the parabolic potential approximation to the Poisson's equation in the cylindrical coordinate system. The threshold-voltage shift in the drain-current model is obtained by solving the nonlinear Poisson-Boltzmann equation for the electrolyte. The simulation results obtained from the proposed drain-current model for the Si-NW field-effect transistor (SiNWFET) agree well with those of the three-dimensional (3D) device simulation, and those from the $\mathrm{Si}-\mathrm{NW}$ pH sensor model also agree with the experimental data.
\end{abstract}

Index Terms - Silicon nanowire, $\mathrm{pH}$ sensor, nonlinear Poisson-Boltzman equation, Poisson's equation, depletion-mode

\section{INTRODUCTION}

Ion-selective field-effect transistors (ISFETs) [1] using silicon nanowires (Si-NWs) have recently attracted considerable attention because they can be applied to many fields of healthcare and life sciences for label-free electrical detection of charged biological species such as pH levels, DNA, proteins, and other biomolecules [2-7]. Furthermore, the use of Si-NWs in $\mathrm{pH}$ sensors has been

Manuscript received Mar. 4, 2014; accepted Jun. 26, 2014 This paper was presented in part at the NANO KOREA 2013. Department of Electrical, Electronic and Control Engineering and IITC, Hankyong National University, Anseong, Korea E-mail : ysyu@hknu.ac.kr demonstrated in literature [2-5]. A compact model for a Si-NW pH sensor has been reported to design and simulate a $\mathrm{Si}-\mathrm{NW} \mathrm{pH}$ sensor system that includes signal amplifying and processing circuits, [8]. A Si-NW fieldeffect transistor (SiNWFET) operating in the inversion mode in Si-NW pH sensors has been modeled [5, 8], but most SiNWFETs have been operated in the depletion mode [2-4]. Therefore, a compact model of a depletionmode SiNWFET in the $\mathrm{pH}$ sensor needs to efficiently simulate the Si-NW $\mathrm{pH}$ sensor and SiNWFET-based integrated systems.

In this paper, we describe a compact analytic model of a depletion-mode $\mathrm{Si}-\mathrm{NW} \mathrm{pH}$ sensor; this drain current model is derived by applying a parabolic potential approximation to Poisson's equation in the cylindrical coordinate system and using the Pao-Sah integral for the Si-NW. Further, the threshold-voltage shift in the draincurrent model is obtained by solving the nonlinear Poisson-Boltzmann equation for the electrolyte. We compare the simulation results obtained from the proposed model with the simulation results of the threedimensional (3D) device simulator as well as the experimental data, to verify the validity of the proposed mode.

\section{Model DeVELOPMENT}

Fig. 1 shows a schematic diagram of a Si-NW pH sensor. The Si-NW in the $\mathrm{pH}$ sensor is covered with a modified surface oxide layer that contains both amino $\left(\mathrm{NH}_{2}\right)$ and silanol $(\mathrm{SiOH})$ groups. $V_{g s}, V_{d}$, and $V_{s}$, represent the voltages at the reference side-gate electrode, drain, and source, respectively. The side-gate can be operated as the reference electrode in the $\mathrm{pH}$ sensor. As 


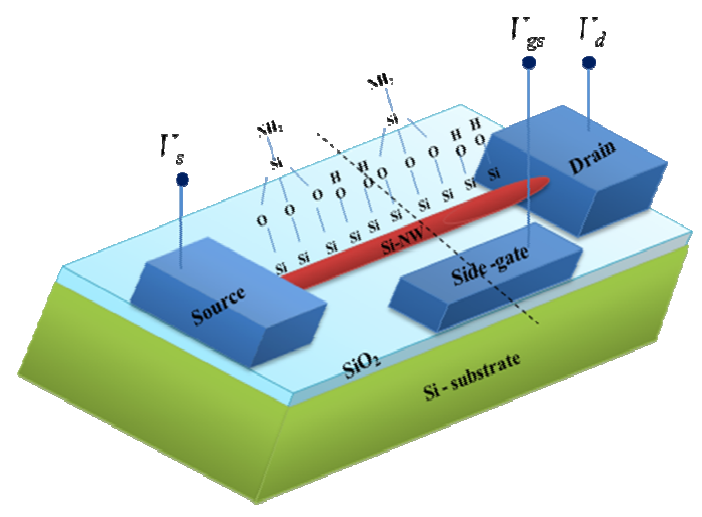

Fig. 1. Schematic of an Si-NW pH sensor.

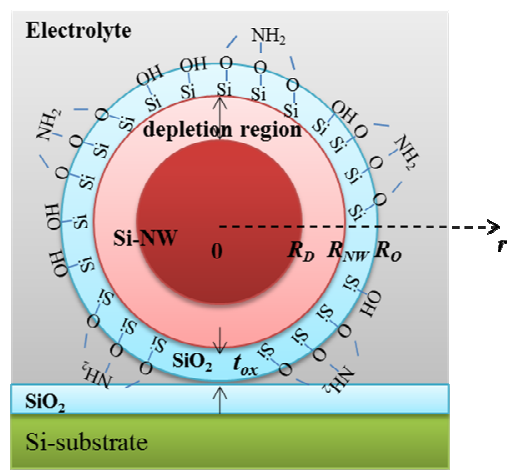

Fig. 2. Cross-section of the Si-NW pH sensor along the dotted line shown in Fig. 1.

shown in Fig. 2, the Si-NW pH sensor contains three regions : a cylindrical $\mathrm{Si}-\mathrm{NW}$ of radius $R_{N W}$, an insulating native oxide layer of thickness $t_{o x}$ around the Si-NW, and an electrolyte that contains the target molecules. Here, $R_{D}$ is the effective radius of the conducting cylindrical region inside the nanowire, which is defined as the difference between the radius and the depletion width, and $R_{O}$ is the distance between the insulator surface and the center of the Si-NW. A semiconductor doped with ptype impurities is used for the Si-NW because most nanowire field-effect-transistor (FET) biosensors have been characterized for the p-type nanowire [2-5]. The metals are used as ohmic contacts for the source and drain.

The potential profile inside the Si-NW in response to the absorbed surface charge is described by the Poisson's equation in cylindrical coordinates for the doped p-type Si-NW:

$$
\frac{1}{r} \frac{d}{d r}\left(r \frac{d \phi}{d r}\right)=\frac{q N_{a}}{\varepsilon_{s i}}\left\{1-\exp \left[-\beta\left(\phi-V_{c h}\right)\right]\right\}
$$

where $\phi$ is the electrostatic channel potential, $\varepsilon_{s i}$ is the silicon dielectric constant, $N_{a}$ is the p-type doping concentration of the $\mathrm{Si}-\mathrm{NW}, r$ is the cylindrical coordinate along the radial direction, $V_{c h}$ is the electron quasi-Fermi potential, $q$ is the electronic charge, and $\beta=$ $q / k_{B} T$ is the inverse of the thermal voltage with the Boltzmann constant $k_{B}$ and the temperature $T$. In the depletion-mode operating regimes of the Si-NW, the minority carrier concentration (electrons) is negligible in comparison with the hole carrier concentration, in the contrast to the our previously reported inversion-mode model [8]. When the boundary conditions [8] are applied to Eq. (1), there is no known analytical solution to Eq. (1), but the parabolic potential approximation was reported as a simple method to represent the potential in the Si-NW [9], and the following equations are obtained by using the method [9]:

$$
\begin{gathered}
V_{g s}-V_{t h}-\eta V_{c h}=-\frac{Q_{m}}{C_{e f f}}-\frac{\eta}{\beta} \ln \left(\frac{Q_{m}}{Q_{0}}\right) \\
-\frac{\eta}{\beta} \ln \left[\left(\frac{Q_{m}}{Q_{\text {dep }}}-1\right) /\left(\exp \left(\frac{\left(Q_{m}-Q_{\text {dep }}\right)}{Q_{0}}\right)-1\right)\right], \\
V_{\text {th }}=E_{\text {ref }}-\Psi_{0}+\chi^{\text {sol }}-\frac{\Phi_{S i}}{q}+\frac{Q_{\text {dep }}}{C_{\text {eff }}} \\
Q_{0}=\frac{2 \varepsilon_{S i}}{\beta R_{N W}}
\end{gathered}
$$

where $1 / C_{\text {eff }}=\eta R_{N W} / 2 \varepsilon_{S i}+1 / C_{o x}, C_{o x}=\varepsilon_{o x} /\left[\left(R_{N W}+\right.\right.$ $\left.\left.t_{o x}\right) \ln \left(1+t_{o x} / R_{N W}\right)\right]$ is the native oxide capacitance [10], $\eta$ is the interface charge parameter [11], $Q_{m}$ is the mobile charge density per unit gate area, $Q_{\text {dep }}=q N_{a} R_{N W} / 2$ is the depletion charge density per unit gate area, $E_{\text {ref }}$ is the constant potential of the reference electrode, $\Psi_{0}$ is the electrolyte/insulator interface potential (sensor surface potential) that is shown to be a function of the solution $\mathrm{pH}, \chi^{\text {sol }}$ is the surface dipole potential of the solvent, and $\Phi_{S i}$ is the workfunction of silicon. $Q_{m}$ in Eq. (2) can be solved by the numerical Newton-Raphson iterative method. Eqs. (2)-(4) are the same as the analytic model for the p-type SiNWFET [12] except for $E_{r e f}, \Psi_{0}$, and $\chi^{\text {sol }}$ in the threshold voltage of Eq. (3), and they are useful to the surrounding-gate nano-scaled devices [9, 12].

When $\mathrm{pH}$ solution is changed, protonation or deprotonation of the modified surface with both $\mathrm{NH}_{2}$ and 
$\mathrm{SiOH}$ groups can change the surface charge density, described by the site-biniding model [13], and then it can change the thickness of the depletion region in the SiNW. Since the surface charge acts as an additional gate, the p-type SiNWFET is depleted if the $\mathrm{pH}$ is decreased (the proton cocntration increased). Using the ISFET model [1] in which the threshold shift due to variation of $\mathrm{pH}$ solution is modeled, the threshold voltage in our model includes the electrolyte/insulator interface potential $\Psi_{0}$ that depend on the $\mathrm{pH}$ value of the electrolyte solution. By solving the nonlinear PoissonBoltzmann equation, $\Psi_{0}$ can be expressed as $[8,10]$

$$
\Psi_{0} \cong \frac{2}{\beta}\left[2.303 \alpha\left|p H-p K_{a}\right|-\frac{\ln \left(I_{0}\right)}{2}+c\right],
$$

where $I_{0}$ is the ion concentration in molar units, $\alpha$ is the dimensionless sensitivity parameter, $p K_{a}=-\log _{10}\left(K_{a}\right), K_{a}$ is the dissociation constant, and

$$
c=\ln \left[\frac{q N_{F}}{\sqrt{2 k_{B} T \varepsilon_{W} N_{a v o}}}\right],
$$

where $N_{F}$ is the density of the surface functionalization groups, $\varepsilon_{W}$ is the dielectric constant of the electrolyte, and $N_{a v o}$ is Avogadro's constant.

An analytical drain-current expression cannot be obtained by integrating the current continuity equation owing to the lack of an analytical integral solution of the last term in Eq. (2). An analytical drain-current model can be obtained by using the following decoupling method of mobile charge [9]: $Q_{m}=Q_{m_{-} \text {dep }}+Q_{m_{-} \text {cor }}$, where $Q_{m_{-} \text {dep }}$ is the mobile charge in the fully-depleted and semi-depleted regions $\left(V_{g s}>V_{F B}\right)$, and $Q_{m_{-} c o r}$ is the complementary mobile charge added to $Q_{m_{\_} d e p}$. $Q_{m_{-} \text {dep }}$ and $Q_{m \text { cor }}$ are independently obtained from the asymptotic behavior of Eq. (2):

$$
\begin{gathered}
V_{g s}-V_{T H_{-} d e p}-\eta V_{c h}=-\frac{Q_{m_{-} d e p}}{C_{e f f}}-\frac{\eta}{\beta} \ln \left(\frac{Q_{m_{-} d e p}}{Q_{0}}\right), \\
V_{g s}-V_{T H_{-} c o r}-\eta V_{c h}=-\frac{Q_{m_{-} c o r}}{C_{o x}-C_{e f f}}-\frac{\eta}{\beta} \ln \left(\frac{Q_{m_{-} c o r}}{Q_{0}}\right),
\end{gathered}
$$

where $V_{T H_{-} \text {dep }}$ and $V_{T H_{-} \text {cor }}$ are the threshold voltages for $Q_{m \_ \text {dep }}$ and $Q_{m_{\text {_cor }}}$ in the depletion-mode Si-NW region, respectively, as follows [12]:

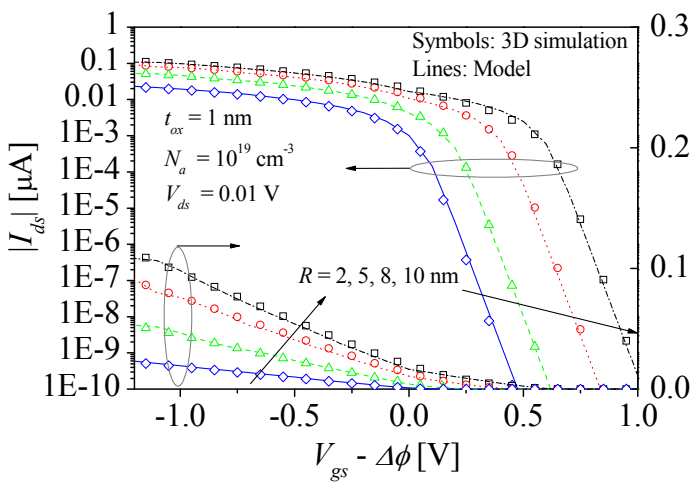

Fig. 3. $I_{d s}-V_{g s}$ characteristics as a function of $R_{N W}$ for an SiNWFET of the $\mathrm{pH}$ sensor.

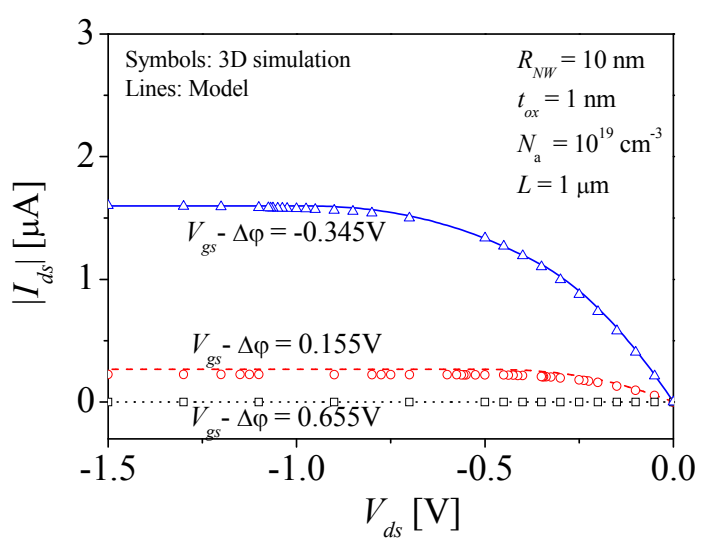

Fig. 4. $I_{\mathrm{ds}}-V_{\mathrm{ds}}$ characteristics as a function of $V_{g s}$ for an SiNWFET of the $\mathrm{pH}$ sensor.

$$
\begin{aligned}
V_{T H_{-} d e p}= & E_{\text {ref }}-\Psi_{0}+\chi^{\text {sol }}-\frac{\Phi_{S i}}{q}+\frac{Q_{\text {dep }}}{C_{e f f}}+\frac{1}{\beta} \ln \left(1-e^{-\frac{Q_{d e p}}{Q_{0}}}\right), \\
V_{T H_{-} c o r} & =E_{\text {ref }}-\Psi_{0}+\chi^{s o l}-\frac{\Phi_{S i}}{q}+\frac{Q_{d e p}}{C_{o x}}+\frac{C_{o x}}{\beta C_{c o r}} \ln \left(\frac{Q_{d e p}}{150 Q_{0}}\right) \\
& -\frac{C_{e f f}}{\beta C_{c o r}}\left[\frac{Q_{d e p}}{Q_{0}}+\ln \left(1-e^{-\frac{Q_{d e p}}{Q_{0}}}\right)\right] .
\end{aligned}
$$

Using the well-known Pao-Sah dual integral method, the drain current can be represented as follows $[9,12]$ :

$$
\begin{aligned}
I_{d s}= & \frac{2 \pi \mu_{e f f} R_{N W}}{L}\left[\frac{Q_{m_{\_} d e p_{-} d}^{2}-Q_{m_{-} d e p_{\_} s}^{2}}{2 \eta C_{e f f}}+\frac{Q_{m_{-} c o r \_d}^{2}-Q_{m_{-} c o r_{-} s}^{2}}{2 \eta\left(C_{o x}-C_{e f f}\right)}\right. \\
& \left.+\frac{1}{\beta}\left(Q_{m_{-} d e p_{-} d}+Q_{m_{-} c o r_{-} d}-Q_{m_{-} d e p_{-} s}-Q_{m_{-} c o r \_s}\right)\right],
\end{aligned}
$$




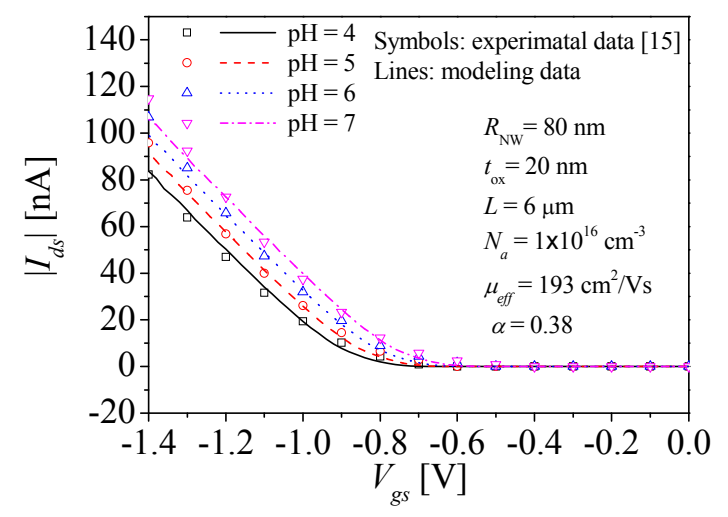

Fig. 5. $\left|I_{\mathrm{ds}}\right|-V_{\mathrm{gs}}$ characteristics at different $\mathrm{pH}$ values; the values were obtained by using the Si-NW pH sensor [15] with $R_{N W}=$ $80 \mathrm{~nm}, t_{o x}=20 \mathrm{~nm}, N_{a}=1 \times 10^{16} \mathrm{~cm}^{-3}, L=6 \mu \mathrm{m}$, and $\mu_{\text {eff }}$ is constant at $193 \mathrm{~cm}^{2} /$ Vs. Symbols and lines denote the experimental data and the model calculations, respectively. $V_{g s}$ is the same as the liquid-gate voltage in [15]

where $\mu_{\text {eff }}$ is the effective hole mobility; $L$ is the effective channel length; $Q_{m_{-} d e \_\_s}$ and $Q_{m_{-} \text {dep } \_d}$ are $Q_{m_{-} d e p}$ at $V_{c h}=0$ $\mathrm{V}$ and $V_{c h}=V_{d s}$, respectively; and $Q_{m_{-} c o r_{-}}$and $Q_{m_{-} \text {cor_d }} d$ are $Q_{m_{c} c o r}$ at $V_{c h}=0 \mathrm{~V}$ and $V_{c h}=V_{d s}$, respectively.

To implement the current model of the Si-NW pH sensor into SmartSpice [14], it can be employed with Verilog-A language on the basis of Eqs. (7)-(11) [15]. The threshold voltage shift due to variation of $\mathrm{pH}$ solution is modeled and implemented with the Verilog-A used in [15]. The SPICE-compatible model of the Si-NW $\mathrm{pH}$ sensor can be used for designing intelligent $\mathrm{pH}$ sensors or micro-nanosystems that are based on Si-NW pH sensors.

\section{MOdel VERIFICATIONS}

To demonstrate the validity of our model, models of the Si-NW pH sensor and its SiNWFET are compared with the experimental data of a Si-NW pH sensor $[2,15]$ and the results simulated from the $3 \mathrm{D}$ device simulator, ATLAS [16], respectively.

Fig. 3 shows the drain-current-side-gate-voltage $\left(I_{d s^{-}}\right.$ $\left.V_{g s}\right)$ characteristics of a depletion-mode p-type SiNWFET at $V_{d s}=0.01 \mathrm{~V}$ as a function of different radii. Fig. 4 shows the drain-current-drain-voltage $\left(I_{d s}-V_{d s}\right)$ characteristics of a depletion-mode p-type SiNWFET at $R_{N W}=10 \mathrm{~nm}$ for different side-gate voltages. The device parameters in this simulation are as follows: $L=1 \mu \mathrm{m}, t_{o x}$ $=1 \mathrm{~nm}, N_{a}=1 \times 10^{19} \mathrm{~cm}^{-3}, \mu_{e f f}=37 \mathrm{~cm}^{2} /(\mathrm{V} \cdot \mathrm{s}), \eta=1$, and

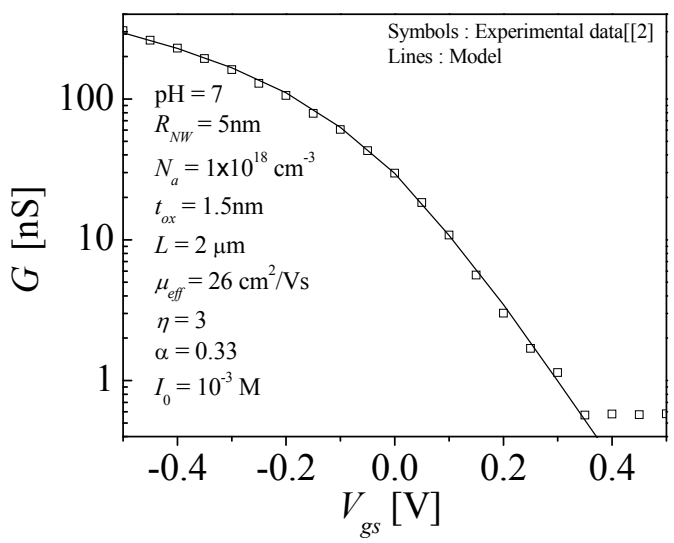

Fig. 6. $d I_{\mathrm{ds}} / d V_{\mathrm{ds}}$ versus $V_{g s}$ at $\mathrm{pH}=7$; the values were obtained by using the Si-NW pH sensor [2] with $R_{N W}=5 \mathrm{~nm}, t_{o x}=1.5$ $\mathrm{nm}, N_{a}=1 \times 10^{18} \mathrm{~cm}^{-3}, L=2 \mu \mathrm{m}$, and $\mu_{\text {eff }}$ is constant at 26 $\mathrm{cm}^{2} /$ Vs. Symbols and lines denote the experimental data and the model calculations, respectively.

$T=300 \mathrm{~K}$. Symbols and lines denote the simulation results of the $3 \mathrm{D}$ device simulator and the proposed model, respectively. The results simulated from the proposed model reproduce those simulated from the 3D device simulator considerably well. Further, They continuously predict the characteristics of the depletionmode p-type SiNWFET in all regions of operation (subthreshold, linear, and saturation regions).

Fig. 5 shows a graph of $I_{d s}$ versus $V_{g s}$ for different $\mathrm{pH}$ values for a test electrolyte used in the Si-NW pH sensor [15]. In this device, $\mathrm{Al}_{2} \mathrm{O}_{3}$ is used instead of the native oxide layer. The device parameters in this simulation are as follows: $L=6 \mu \mathrm{m}, R_{N W}=80 \mathrm{~nm}, t_{o x}=20 \mathrm{~nm}, N_{a}=$ $1 \times 10^{16} \mathrm{~cm}^{-3}$, and $T=300 \mathrm{~K}$. The extracted parameters are the mobility $\mu_{\text {eff }}=193 \mathrm{~cm}^{2} /(\mathrm{V} \cdot \mathrm{s}), \eta=1, \alpha=0.38, I_{0}=10^{-}$ ${ }^{3} \mathrm{M}, \chi^{\text {sol }}=0.003 \mathrm{~V}$ [17], and $E_{\text {ref }}=4.055 \mathrm{~V}$. The device parameters are similar to those extracted from Verilog-A model in [15]. As shown in Fig. 5, the model calculations (lines) agree well with the experimental data (symbols).

Fig. 6 shows a graph of the zero-bias differential conductance versus $V_{g s}$ at $\mathrm{pH}=7$ for a test electrolyte used in the Si-NW pH sensor [2]. The device parameters in this simulation are as follows: $L=2 \mu \mathrm{m}, R_{N W}=5 \mathrm{~nm}$, $t_{o x}=1.5 \mathrm{~nm}, N_{a}=1 \times 10^{18} \mathrm{~cm}^{-3}$, and $T=300 \mathrm{~K}$. The extracted parameters are the mobility $\mu_{\text {eff }}=26 \mathrm{~cm}^{2} /(\mathrm{V} \cdot \mathrm{s})$, $\eta=3, \alpha=0.33, I_{0}=10^{-3} \mathrm{M}, \chi^{\text {sol }}=0.003 \mathrm{~V}[17]$, and $E_{\text {ref }}$ $=4.754 \mathrm{~V}$. Fig. 7 shows the graph of the zero-bias differential conductance versus $\mathrm{pH}$ for three values of $V_{g s}$ for the same device parameters used to plot Fig. 6. The 


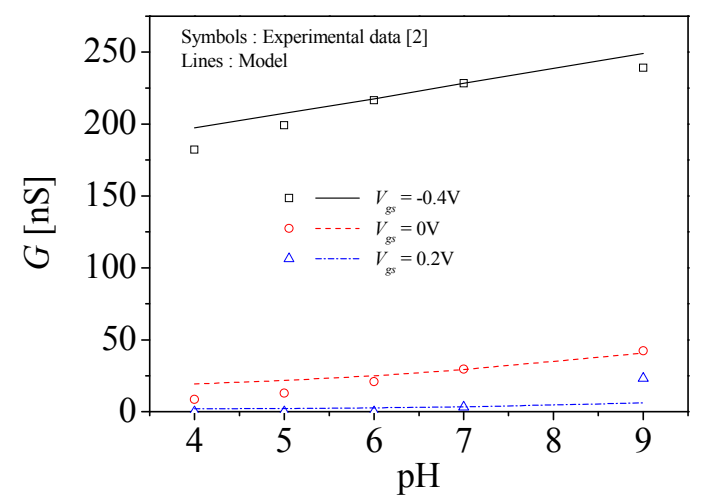

Fig. 7. $d I_{\mathrm{ds}} / d V_{\mathrm{ds}}$ versus $\mathrm{pH}$ as a function of $V_{\mathrm{gs}}$; the values were obtained by using the Si-NW pH sensor [2] with the same parameters used to plot Fig. 6. Symbols and lines denote the experimental data and the model calculations, respectively.

extracted parameters were the same as those shown in Fig. 6. As shown in Figs. 6 and 7, the model calculations (lines) agree well with the experimental data (symbols). As the $\mathrm{pH}$ increases, the zero-bias conductance increases in the depletion-mode Si-NW pH sensor, as shown in Fig. 7, whereas it decreases in the inversion-mode SiNW pH sensor $[5,8]$. Because the resulting pH-dependent electrical surface charge of the insulator leads to the modulation of the channel conductance of the Si-NW pH sensor, it is possible to determine the $\mathrm{pH}$ of the test solution by quantitatively measuring the changes in the channel conductance.

\section{CONCLUSIONS}

In conclusion, we presented a compact analytic model of a depletion-mode Si-NW pH sensor. This drain current model was obtained by using the Pao-Sah integral and the continuous charge-based model which was derived by applying the parabolic potential approximation to Poisson's equation in the cylindrical coordinate system. The threshold-voltage shift in the current model was obtained by solving the nonlinear Poisson-Boltzmann equation in the electrolyte. The simulation results obtained from the proposed model agree well with the simulation results of the $3 \mathrm{D}$ device simulation and the experimental data. Because advanced physical effects such as short-channel and quantum effects are not included in the proposed model, it is necessary to extend the model by including these effects. The proposed model can easily be applied to commercially available electronic design automation (EDA) tools that are commonly used for integrated circuit design and simulations.

\section{ACKNOWLEDGMENTS}

This research was supported by the Basic Science Research Program through the National Research Foundation of Korea (NRF) funded by the Ministry of Education (NRF-2011-0009829).

\section{REFERENCES}

[1] P. Bergveld, "Thirty years of ISFETOLOGY: What happened in the past 30 years and what may happen in the next 30 years," Sens. Actuators B: Chem., Vol. 88, No. 1, pp. 1-20, 2003.

[2] X. P. A. Gao, G. Zheng, and C. M. Lieber, "Subthreshold regime has the optimal sensitivity for nanowire FET biosensors," Nano Lett., Vol. 10, No. 2, pp. 547-552, 2010.

[3] O. Knopfmacher, A. Tarasov, W. Fu, M. Wipf, B. Niesen, M. Calame, and C. Schönenberger, "Nernst limit in dual-gated Si-nanowire FET sensors," Nano Lett., Vol. 10, No. 6, pp. 2268-2274, 2010.

[4] S. Zafar, C. D'Emic, A. Afzali, B. Fletcher, Y. Zhu and $\mathrm{T}$. Ning, "Optimization of $\mathrm{pH}$ sensing using silicon nanowire field effect transistors with $\mathrm{HfO} 2$ as the sensing surface," Nanotechnol., Vol. 22, No. 40, pp. 405501, 2011.

[5] Y. Chen, X. Wang, S. Erramilli, P. Mohanty, and A. Kalinowski, "Silicon-based nanoelectronic fieldeffect $\mathrm{pH}$ sensor with local gate control," Appl. Phys. Lett., Vol. 89, p. 223512, 2006.

[6] Y. L. Bunimovich, Y. S. Shin, W. Yeo, M. Amori, G. Kwong, and J. R. Heath, "Quantitative real-time measurements of DNA hybridization with alkylated nonoxidized silicon nanowires in electrolyte solution," J. Amer. Chem. Soc., Vol. 128, No. 50, pp. 16323-16331, 2006.

[7] J. Yao, H. Yan, and C.M. Lieber, "A nanoscale combing technique for the large-scale assembly of highly aligned nanowires," Nature Nanotechnol., Vol. 8, No. 5, pp. 329-335, 2013.

[8] Y. S. Yu and S. W. Hwang, "Analytic Model of A Silicon Nanowire pH Sensor," J. Nanosicence 
Nanotechnogy, Vol. 11, No. 12, pp. 10809-10812, 2011.

[9] J. P. Duarte, S. J. Choi, D. I. Moon, and Y. K. Choi, "A nonpiecewise model for long-channel junctionless cylindrical nanowire FETs," IEEE Electron Device Lett., Vol. 33, No. 2, pp. 155-157, Feb. 2012.

[10] P. R. Nair and M. A. Alam, "Screening limited response of nanobiosensor," Nano Lett., Vol. 8, No. 5, pp. 1281-1285, 2008.

[11] Y. S. Yu, N. Cho, S. W. Hwang, and D. Ahn, "Implicit continuous current-voltage model for surrounding-gate metal-oxide-semiconductor fieldeffect transistors (SGMOSFETs) including interface traps," IEEE Trans. Electron Devices, Vol. 58, No. 8, pp. 2520-2524, 2011.

[12] Y. S. Yu, "A unified analytical current model for $\mathrm{N}$ - and P-type accumulation-mode (junctionless) surrounding-gate nanowire FETs," IEEE Trans. Electron Devices, Vol. 61, No. 8, pp.3007-3010, 2014.

[13] D. E. Yates, S. Levine, T. W. Healy, "Site-binding model of the electrical double layer at the oxide/water interface," J. Chem. Soc. Faraday Trans. 1, Vol. 70, pp. 1807-1818, 1974.

[14] Silvaco, Inc. SMARTSPICE Users' Manual. (2012). [Online]. Available: http://www.silvaco.com.

[15] P. Livi, K. Bedner, A. Tarasov, M. Wipf, Y. Chen, C. Schönenberger, A. Hierlemann, "A Verilog-A model for silicon nanowire biosensors: from theory to verification," Sensors and Actuators B: Chemical, Vol. 179, pp. 293-300, 2013.

[16] ATLAS ver. 5. 18. 3. R Manual, Silvaco International, Santa Clara, CA (2012).

[17] G. Massobrio, M. Grattarola, G. Mattioli, and F. Mattioli, "ISFET-based Biosensor Modeling with SPICE," Sensors and Actuators B: Chemical, Vol. 1, pp. 401-407, 1990.

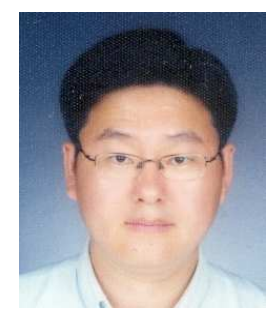

Yun Seop Yu received the B.S., M.S., and Ph. D. degrees in electronics engineering from Korea University, Seoul, Korea, in 1995, 1997, and 2001, respectively. From 2001 to 2002, he worked as a guest researcher at the Electronics and Electrical Engineering Laboratory in NIST, Gaithersburg, MD. He is now a full Professor with the Department of Electrical, Electronic and Control Engineering at Hankyong National University, Anseong, Korea. His main research interests are in modeling various nanodevices for efficient circuit simulation, and future memory, logic, and sensor designs using those devices. $\mathrm{He}$ is also interested in the fabrication and characterization of various nano devices, as well as their future applications in memory, logic, and sensors. 